\title{
愈创木烷型倍半萜类天然产物及其类似物的全合成研究进展
}

\author{
卢勇平祁伟鹏翁国栋张兴贤* \\ (浙江工业大学药学院 浙江杭州 310032)
}

\begin{abstract}
摘要 综述了愈创木烷型倍半萜类天然产物及其类似物的全合成研究进展. 主要包括以卡宾羰基叶立德环化串联反 应、烯烃复分解环化反应、分子内羟醛缩合反应等作为关键反应的方法来构筑该类天然产物的基本骨架.

关键词 愈创木烷型倍半萜; 天然产物; 全合成

\section{Progresses in Total Synthesis of Guaiane Sesquiterpenes and Their Analogues}

\author{
Lu, Yongping Qi, Weipeng Weng, Guodong Zhang, Xingxian* \\ (College of Pharmaceutical Sciences, Zhejiang University of Technology, Hangzhou, Zhejiang 310032)
}

\begin{abstract}
The recent progresses in the total synthesis of guaiane sesquiterpenes and their analogues are reviewed, which include the carbene cyclization-cycloaddition cascade reaction, olefin ring closing metathesis reaction, intramolecular aldol reaction as the key steps to construct basic ring system of these natural products.

Keywords guaiane sesquiterpenes; natural products; total synthesis
\end{abstract}

愈创木烷型倍半萜(guaianoid)是自然界中存在的一 类重要天然产物, 已经分离鉴定的愈创木烷型倍半萜类 天然产物有数百种 ${ }^{1]}$. 从中药姜黄中分离得到氧杂愈创 木烷型倍半萜莪术醇(curcumol), 临床用于治疗宫颈癌, 具有无致突变性和不良反应少的特点 ${ }^{[2]}$, 是高效、低毒、 安全可靠的抗肿瘤药物, 而且对多种疾病有效 ${ }^{[3 \sim 5]}$. 从 金钱松根部分离得到愈创木烷型倍半萜内酯土槿皮酸 (pseudolaric acid) ${ }^{[6]}$, 对多种癌细胞具有显著的抑制作 用 ${ }^{[7]} .2008$ 年底美国国立癌症研究所(NCI)的 Beutler 小 组 ${ }^{[8]}$ 从生长于东非坦桑尼亚及津巴布韦的大戟属植物 Phyllanthus engleri 的树皮和根皮提取液中分离得到氧 杂愈创木烷型倍半萜天然产物(一)-englerin $\mathrm{A}^{[9]}$, 其良好 的生物活性, 潜在的成药性和独特的化学结构引起了世 界范围内有机合成化学家的广泛关注. 图 1 列举了几种 具有代表性的该类天然产物, 其中不少都表现出很好的 生物活性.

\section{1 愈创木烷型倍半萜类天然产物及其类似物的 全合成概述}

愈创木烷型倍半萜类天然产物骨架中含有一个典 型的 $[5,7]$ 并环结构单元, 其中七元环通过半缩酮的氧 桥, 形成了一个五元环和六元环. 同时分子中具有多个 连续的手性中心, 其中 2 个为杂季碳, 这使得其不对称 全合成工作充满了挑战, 一直是有机化学家特别是合成 化学家颇感兴趣的课题之一, 许多全合成工作都围绕着 其碳骨架的建立而展开 ${ }^{[10]}$.

\section{1 以卡宾羰基叶立德环化串联反应为关键反应的方 法}

卡宾羰基叶立德环化串联 $(\mathrm{CCCC})$ 反应在天然产物 的合成中有着广泛的应用 ${ }^{[11]}$. 重氮基团与羰基在金属催 化剂催化下环化形成一个以氧原子为中心 1,3 -偶极体 系，再与烯基发生一个串联反应(Scheme 1). 此反应可 以构建愈创木烷型倍半萜类天然产物的基本骨架. 基于 此, 许多研究小组利用 $\mathrm{CCCC}$ 反应来合成愈创木烷型倍 半萜类天然产物及其类似物.

\footnotetext{
* E-mail: zhangxx@zjut.edu.cn

Received December 27, 2014; revised March 11, 2015; published online March 24, 2015.

Project supported by the National Natural Science Foundation of China (Nos. 21372203, 21272076)

国家自然科学基金(Nos. 21372203, 21272076)资助项目.
} 


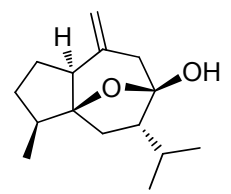

(-)-curcumol (1)

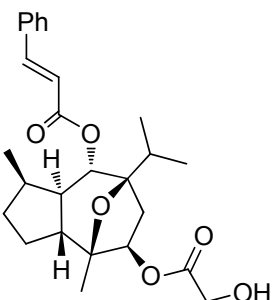

(-)-englerin A (4)

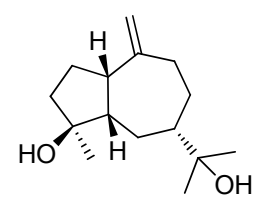

pleocarpenene (2)

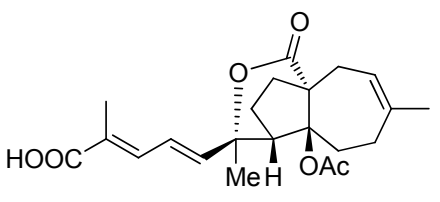

pseudolaric acid (5)

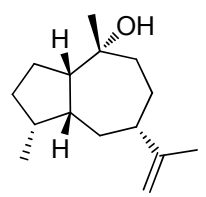

pogostol (3)

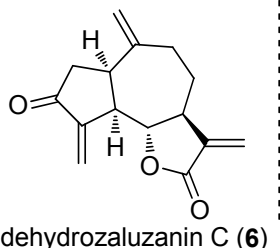

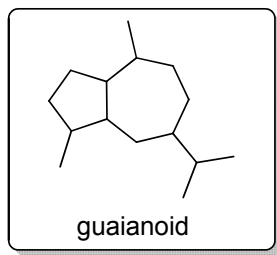

图 1 代表性愈创木烷型天然产物

Figure 1 The typical guaianoid natural products

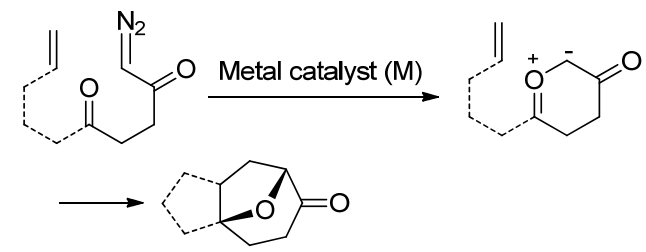

图式1 卡宾羰基叶立德环化串联反应

Scheme 1 Carbene carbonyl ylid cyclization cycloaddition

2003 年 Chiu 小组 ${ }^{[12]}$ 首次报道了土槿皮酸(pseudolaric acid)的全合成工作, 并验证了该天然产物的分子结 构. 以 3-氯-2-氯甲基丙烯(10)为原料, 经 4 步反应得到 中间体 11, 然后通过 Evens 不对称羟醛缩合反应建立中 间体 12 的绝对构型, 与 Normant 试剂加成延长碳链得到 关环前体化合物 13, 然后经金属铑催化的 CCCC 反应一 步构建 pseudolaric acid 基本骨架, 最终完成该天然产物 pseudolaric acid (5)的全合成, 合成路线如 Scheme 2 所 示.

2010 年 Maier 小组 ${ }^{[13]}$ 利用 CCCC 反应对 englerin A 骨架的合成开展了探索性研究, 在合成手性五元环骨架 17 的基础上, 通过金属铑催化的 $\mathrm{CCCC}$ 反应建立 englerin A 的氧杂 $[5,7]$ 并环结构. 采用 Scheme 3 所示的 合成路线, 以 $(R)-(-)$-香芹酮 $(15)$ 为起始原料, 经环氧 化、Favorskii 重排、脱保护等 5 步反应制备五元环化合 物 16, 再通过 Barton-McCombie 反应脱除羟基、氢化铝 锂还原、Parikh-Doering 氧化、DBU 诱导醛基差向异构 化等反应得到关环前体化合物 $\mathbf{1 7}$, 然后在金属铑催化 下经 CCCC 反应构建 englerin A 三环骨架, 遗憾的是氧 桥的立体构型与 englerin A 相反.

\section{3 以烯烃复分解环化反应为关键反应的方法}

烯烃复分解环化(RCM) 反应是通过非环二烯化合 物来构筑不同大小环系化合物的一种最直接、最有效的

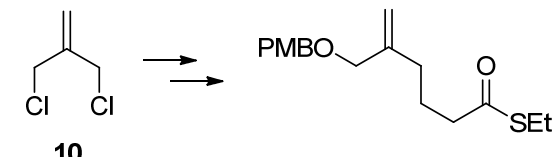

10

11

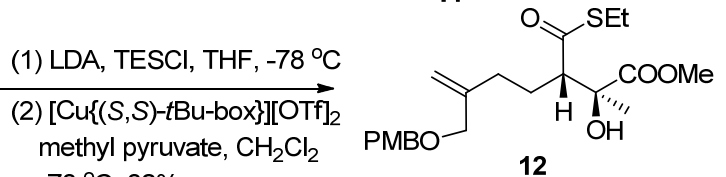
$-78^{\circ} \mathrm{C}, 88 \%$ ee
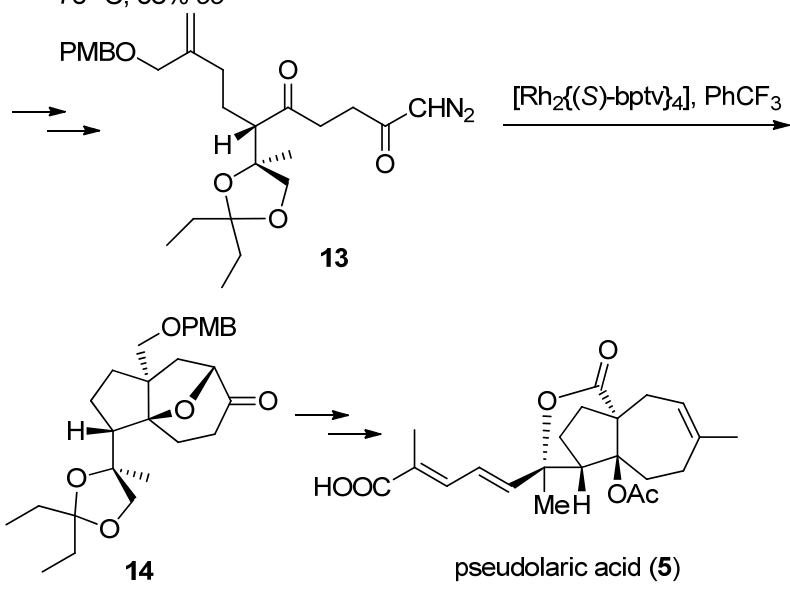

图式 2 土槿皮酸的 Pauline 合成路线

Scheme 2 Pauline's synthesis of pseudolaric acid

合成方法之一 ${ }^{[14]}$. 近几年来的研究表明, RCM 反应在构 筑不同大小的环系类化合物方面也非常有效.

2009 年底, Christmann 小组 ${ }^{[15]}$ 首次报道了 englerin A 的全合成工作。其基本骨架的构建采用了第二代 Grubbs 催化剂催化的 RCM 反应. 以(一)-荆芥内酯 (20)为起始原料, 经环氧内酯重排、Barbier 反应、缩醛 保护、DBU 催化的差向异构化、Witing 反应得到环化 前体二烯 21, 再通过 RCM 建立该天然产物的基本骨架, 从而为(一)-englerin $\mathrm{A}$ 的不对称全合成奠定了很好的基 础，其合成路线如 Scheme 4 所示. 
<smiles>C=CC1(C)CC=C(C)C(=O)C1</smiles>

15

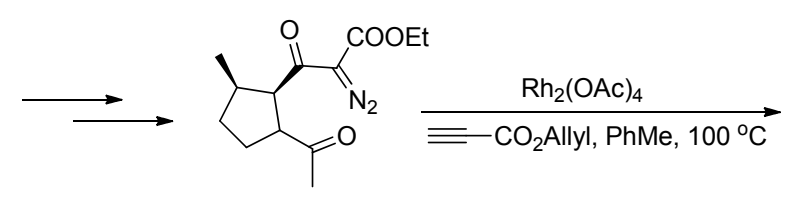

17

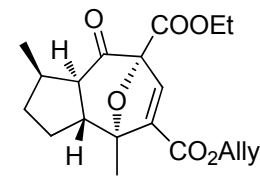

18<smiles>C=C(C)[C@H]1C[C@@H](O)[C@H](C)[C@@H]1C(C)=O</smiles>

16

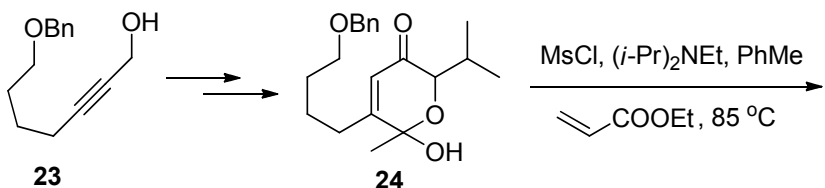

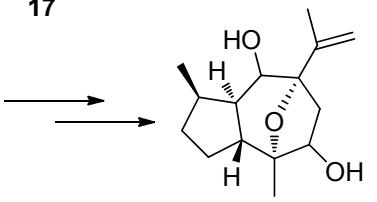

19
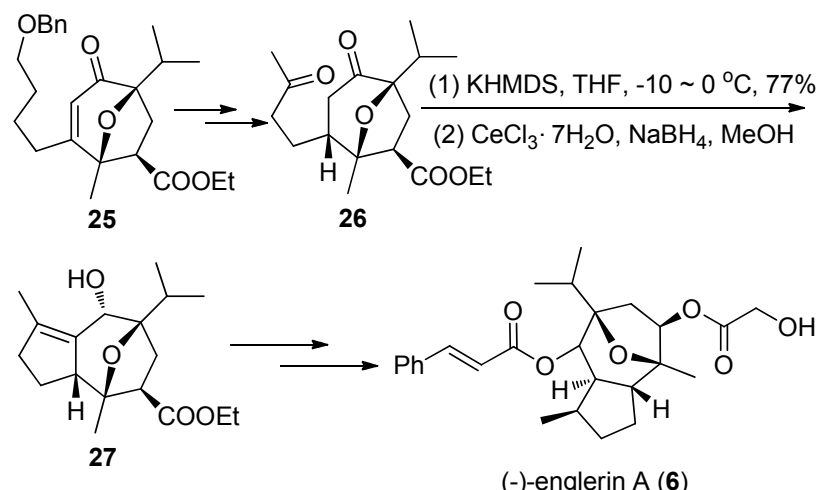

图式 3 englerin A 类似物的 Maier 合成路线

Scheme 3 Maier's synthesis of englerin A analogues

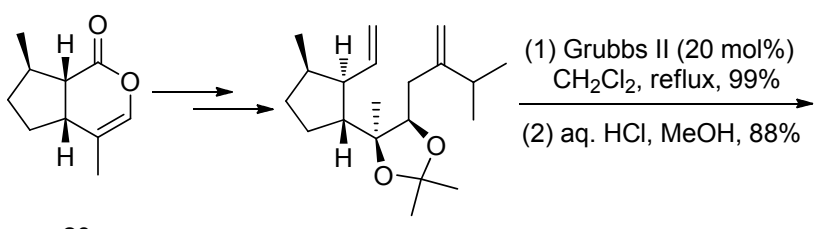

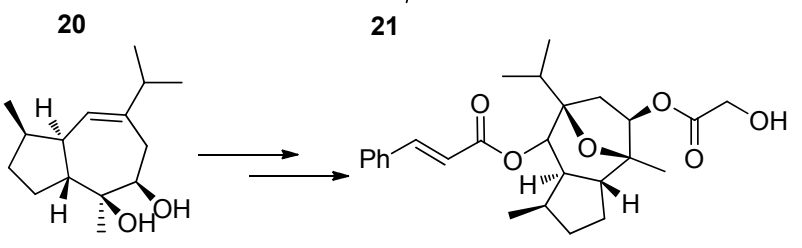

22

(-)-englerin A (6)

图式4 (一)-englerin A的Theodorakis合成路线

Scheme 4 Christmann's synthesis of (-)-englerin A

\section{3 以分子内羟醛缩合反应为关键反应的方法}

在建立碳一碳键的合成方法中, 䤊醇缩合反应是最 直接、最有效的一类化学转化之一, 它被广泛应用于复 杂分子的合成和光学活性小分子砌块的制备 ${ }^{[16]}$.

Nicolaou 小组 ${ }^{[17]}$ 以炔醇 23 为起始原料, 经过碘化、 甲酰化、格氏加成、Achmatowicz 重排等反应得到 3-氧 代吡喃内盐 24, 再与丙烯酸乙酯发生 $[5+2]$ 环加成反应 得到七元环化合物 $\mathbf{2 5}$, 经脱保护、氧化消除、Wacker 氧 化得到关环前体二酮 26, 通过分子内羟醛缩合反应构 筑该天然产物的基本骨架，从而完成了 englerin $\mathrm{A}$ 全合 成, 其合成路线如 Scheme 5 所示.

Theodorakis 小组 ${ }^{[18]}$ 也报道了采用分子内羟醛缩合 反应构筑(一)-englerin A 基本骨架的合成方法, 如 Scheme 6 所示. 首先用甲基异丙基呋喃(28)在辛酸铑催 化下与手性重氮酯 $\mathbf{2 9}$ 反应构建七元环化合物 $\mathbf{3 0}$, 然后
图式 5 (一)-englerin A 的 Nicolaou 合成路线

Scheme 5 Nicolaou's synthesis of (-)-englerin A

用 DIBAL-H 还原、路易斯酸催化重排、Rubottom 氧化 等反应得到关环前体二酮 31, 接着以分子内羟醛缩合 反应合环制备氧杂三环体系，建立该天然产物的骨架， 从而完成其全合成.

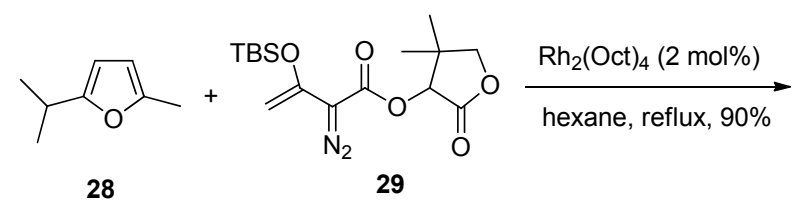

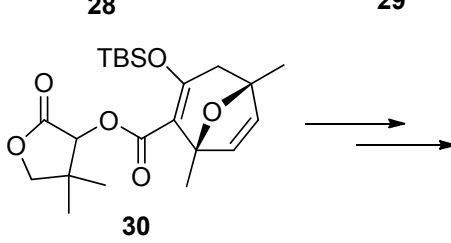

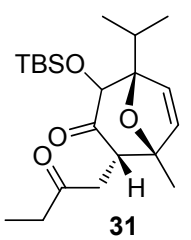

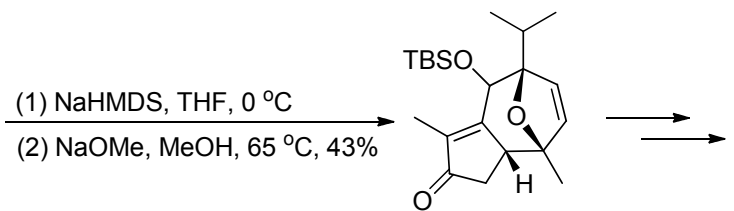<smiles>CC1CCCC2(C1)OC1(OC(=O)/C=C/c3ccccc3)C(C)(C)C(OC(=O)CO)CC21C(C)C</smiles>

(-)-englerin A (6)

图式 $6(-)$-englerin A 的 Theodorakis 合成路线

Scheme 6 Theodorakis's synthesis of (-)-englerin A

以上用分子内的羟醛缩合反应合成愈创木烷型倍 半萜类天然产物的例子都是先建立目标分子的碳骨架, 然后通过分子内的羟醛缩合反应来构筑 5,7-元环核心结 构. 


\section{4 以金 $(I)$ 催化 $[2+2+2]$ 环加成串联反应为关键反应 的方法}

最近，马大为教授 ${ }^{[19]}$ 报道了(-)-englerin $\mathrm{A}$ 的不对 称全合成工作, 采用金(I)催化 $[2+2+2]$ 环加成串联反 应为关键步骤, 一步构筑(一)-englerin A 基本骨架, 合 成路线如 Scheme 7 所示. 以 $(R)-(+)$-香茅醛 $(\mathbf{3 3})$ 为原料, 经溴化、消除、Riley 氧化和 IBX 氧化在烯丙位引入羰 基、与烯醇硼酸酯发生不对称羟醛缩合反应，以较高的 收率得到环化前体化合物 34, 然后在 $10 \mathrm{~mol} \% \mathrm{AuCl}$ 催 化下发生 $[2+2+2]$ 环加成串联反应, 一步构建 (一)-englerin A 氧杂 5,7-元环基本骨架, 从而完成天然产 物(一)-englerin A 的不对称全合成.

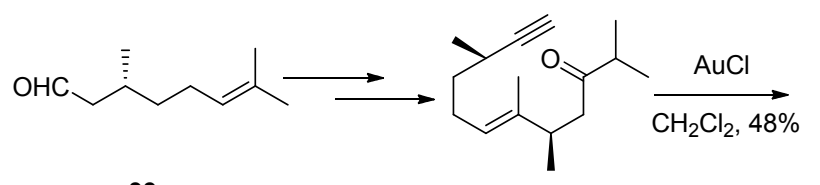

33

34

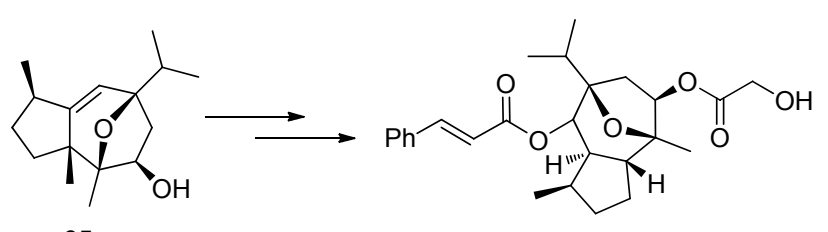

35

(-)-englerin A (6)

图式 7 (一)-englerin A 的马大为合成路线

Scheme 7 Ma's synthesis of (-)-englerin A

2006 年, Echavarren 小组 ${ }^{[20]}$ 报道了 englerin $\mathrm{A}$ 的类 似物 $(+)$-orientalol $\mathrm{F}$ 的全合成工作, 采用了 1,6-烯炔在 金(I)催化下发生 $[2+2+2]$ 环加成品联反应成功构建 5,7 元环及氧桥键, 合成路线如 Scheme 8 所示. 随后在此研 究的基础上, 以香叶醇为起始原料 ${ }^{[21]}$, 完成了 (一)-englerin A 的全合成, 合成路线如 Scheme 9 所示. 香叶醇经 Sharpless 不对称环氧化、伯醇氧化、丁基锂 消除得到炔丙醇 $\mathbf{4 0}$, 在 TES 保护、氧化裂解、Wittig 反 应后生成反式烯烃 41 , 从而在手性磷酰胺的存在下与 烯醇硅醚进行 Denmark 不对称 aldol 反应生成环化前体 42, 之后在金 $(\mathrm{I})$ 催化下发生 $[2+2+2]$ 环加成串联反应 成功构建氧桥 $[5,7$ 元]核心骨架, 最终完成(一)-englerin $\mathrm{A}$ 的不对称全合成.

\section{5 其他方法}

2006 年 Snapper 小组 ${ }^{[22]}$ 报道了天然产物 plecarpenene 和 pleocapenone 全合成. 以 $\alpha$-吡喃酮 44 为 起始原料, 与 $\mathrm{Fe}_{2}(\mathrm{CO})_{9}$ 发生光化学电子环化反应生成铁 环丁烯甲酯络合物 $\mathbf{4 5}$, 然后经 DIBAL-H 还原、 $\mathrm{MnO}_{2}$ 氧化、高烯丙基溴化镁加成得到中间体 46, 再经 CBS 不对称还原、 RCM 反应得到化合物 $\mathbf{4 7}$, 化合物 47 在

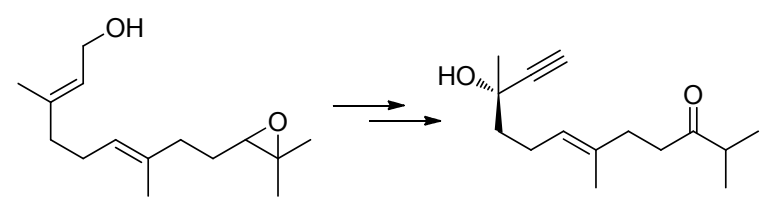

(+)-epoxyfarnesol (36)

37
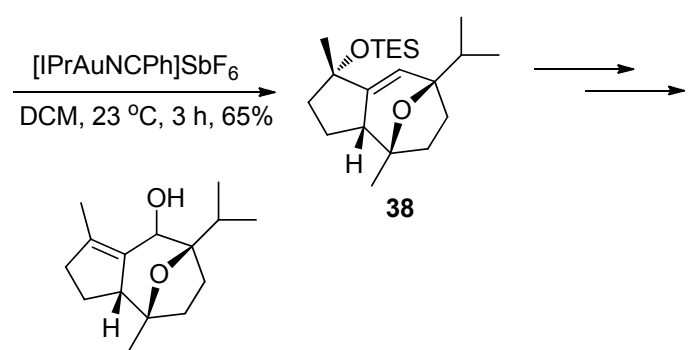

38

(+)-orientalol F (39)

图式 $8(+)$-orientalol F 的 Echavarren 合成路线 Scheme 8 Echavarren 's synthesis of $(+)$-orientalol F<smiles>C#C[C@@](C)(CCC#CC#C[C@](C)(O)CCC=C(C)C)CC/C=C(\C)C=O</smiles>

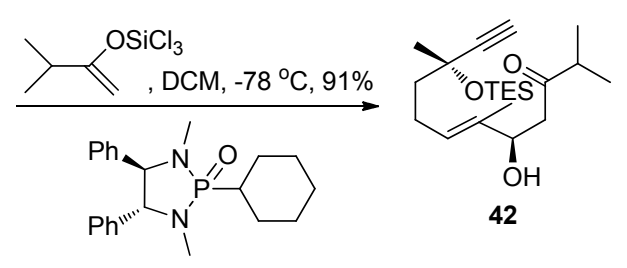

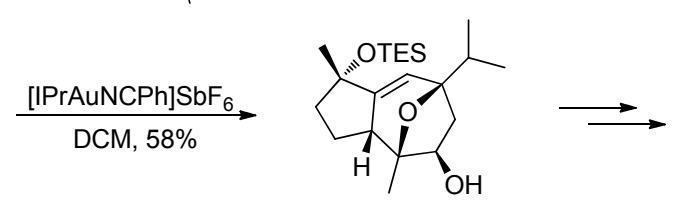

43

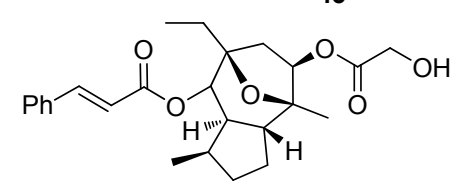

(-)-englerin A (6)

图式 9 (-)-englerin A 的 Echavarren 合成路线

Scheme 9 Echavarren 's synthesis of ( - )-englerin A

CAN 催化下发生分子内的环丁烯氧化环合得到一对非 对映异构体 48a 和 48b. 该非对映异构体经酯基还原、 差基保护、 $\mathrm{Cu}(\mathrm{acac})_{2}$ 催化双键环丙烷化反应得到稠环化 合物 40, 然后经 Swern 氧化、格式加成建立分子碳骨架, 最后在 DBU 催化下通过高温热重排反应构建 5, 7 元环 基本骨架，进而完成了 plecarpenene 和 pleocapenone 的 全合成(Scheme 10). 
<smiles></smiles>

44

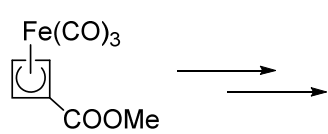

45
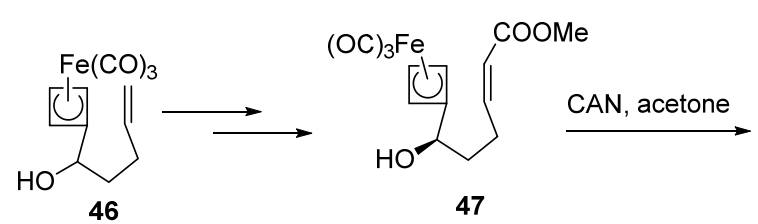<smiles>COC(=O)[C@H]1[C@H]2C=C[C@@H]3[C@@H](O)CC[C@H]1[C@@H]23</smiles>

48a

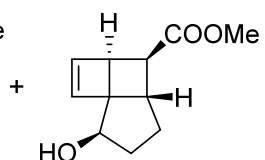

48b
(1) $\mathrm{LAH}, \mathrm{THF}$ (2) TIPSCI, DMAP

(3) EDA, $\mathrm{Cu}(\mathrm{acac})_{2}$

(1) $(\mathrm{COCl})_{2}, \mathrm{DMSO}$ $\mathrm{Et}_{3} \mathrm{~N}, \mathrm{THF},-62^{\circ} \mathrm{C}$

(2) $\mathrm{MeMgCl},-78^{\circ} \mathrm{C}$

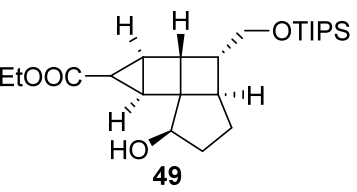

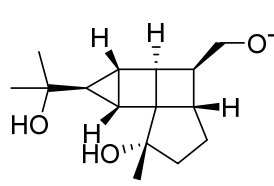

50

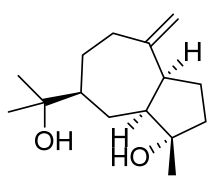

pleocarpenene (2)
(1) $\mathrm{O}_{3}, \mathrm{MeOH},-78^{\circ} \mathrm{C}$

(2) DMS, r.t.

(3) $\mathrm{NaOMe}$

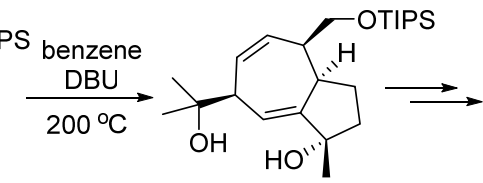

51

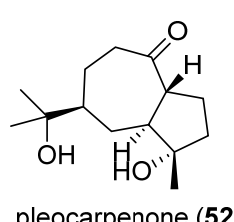

图式 10 plecarpenene 和 pleocapenone 的 Snapper 合成路线 Scheme 10 Snapper's synthesis of plecarpenene and pleocapenone

2010 年 Srikrishna 小组 ${ }^{[23]}$ 报道了 11-hydroxyguaiadienes (57)的全合成, 其关键骨架 5, 7 元环的构建采用 了 ene-aldol 反应, 合成路线如 Scheme 11 所示. 从 $(R)-$ 柠檬烯(53)出发, 经臭氧化、重排、还原、溴化得到化 合物 54, 再通过亲核取代、还原、IBX 氧化得到了环化 前体 55, 在 $\mathrm{BF}_{3} \cdot \mathrm{Et}_{2} \mathrm{O}$ 催化下经 ene-aldol 反应构建 5,7 元环基本骨架，最终完成 11-hydroxyguaiadienes 全合成.

Lee 小组 ${ }^{[24]}$ 报道了 clavukerin A 全合成, 采用如 Scheme 12 中低价钛诱导的分子内二羰基化合物的偶合 反应为关键步骤来构建该天然产物骨架. 从 $(R)$-氧化柠 檬烯 68 出发, 在酸性条件下环氧开环生成邻位二醇, 经 $\mathrm{NaIO}_{4}$ 氧化、分子内羟醛缩合得到环戊烯衍生物 69, 后 经多步反应得到关环前体二醛 70, 最后通过低价钛诱 导的分子内二羰基化合物的偶合反应完成 clavukerin A 全合成.

林国强研究小组 ${ }^{[25]}$ 采用 Heck 偶联反应来合成

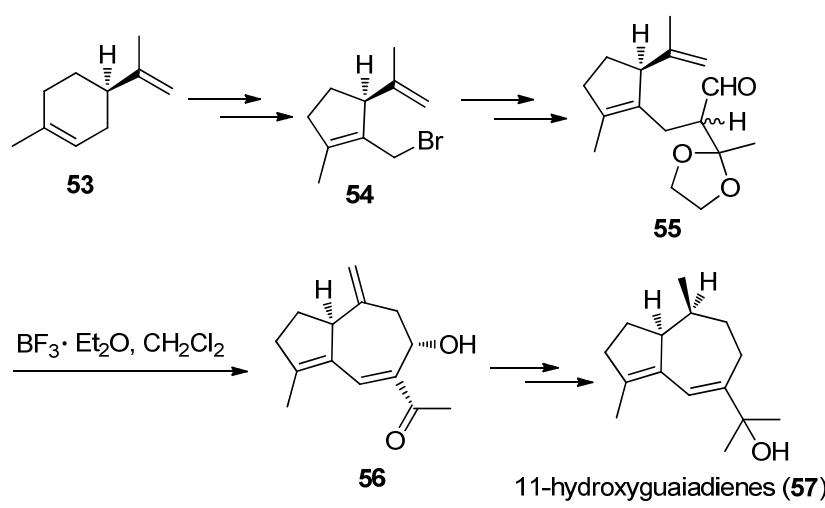

图式 11 11-hydroxyguaiadienes 的 Srikrishna 合成路线

Scheme 11 Srikrishna's synthesis of 11-hydroxyguaiadienes

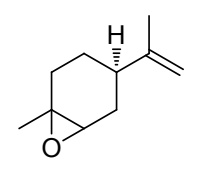

68

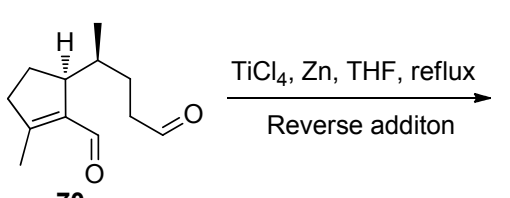

70

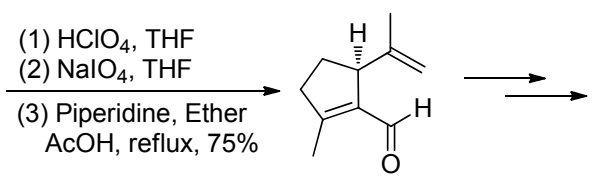

69

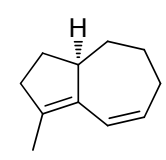

(-)-clavukerin A (71)
图式 12 clavukerin A 的 Lee 合成路线

Scheme 12 Lee's synthesis of clavukerin A

(一)-englerin A 基本骨架，合成路线如 Scheme 13 所示. 利用手性有机小分子催化呋喃化合物 58 和烯醇硅醚 59 发生[4+3]环加成反应得到手性 8-氧代-双环[3.2.1]辛烯 片段 60, 然后经碳链的延长得到环化前体 61, 再在碱性 条件下发生 Heck 偶联反应得到基本骨架 62 , 最终完成 (一)-englerin A 的全合成.

Maier 小组 ${ }^{[26]}$ 利用 CCCC 反应对 englerin A 的合成 开展研究之后，随后在 2011 年 3 月报道了策略完全不同 的方法，完成了对(一)-9-deoxy-englerin A 的全合成，其 核心步骤采用颇具特色的跨环环氧开环反应来构建 5,7 元环(合成路线如 Scheme 14 所示). 起始原料为(一)-异 胡薄荷醇(63), 通过 PCC 氧化、乙烯基格式试剂加成、 oxy-Cope 重排为环癸烯酮、 $m$-CPBA 氧化成环氧化合物 64, 碱催化下分子内环氧开环得到 5,7 元环 65. 同时该 小组发现了羟基酮 65 和其半缩醛 $65 \mathrm{a}$ 形式存在自发平 衡，但将混合物的差基保护后，两者就容易分离，同时 半缩醛 66a 水解得到化合物 65a. 化合物 66 经多步转化 最终得到(一)-englerin A 衍生物(一)-9-deoxy-englerin A (67).

Chain 小组 ${ }^{[27]}$ 将 $\mathrm{SmI}_{2}$ 催化的还原偶联反应成功地应 用于 englerin A 的全合成, 非常巧妙、高效地完成了该 分子骨架的构建. 以 3-呋喃酮 72 和手性环戊烯醛(73) 

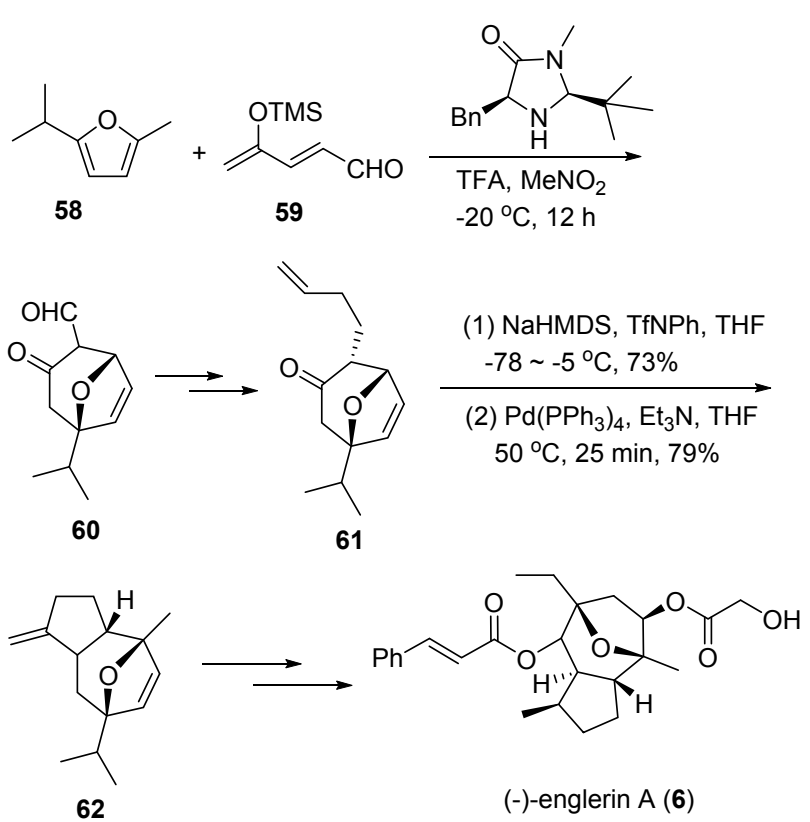

图式 13 (一)-englerin A 的林国强合成路线 Scheme 13 Lin's synthesis of ( - )-englerin A<smiles>C=C(C)[C@@H]1CCC(C)C[C@H]1O</smiles>

63

64

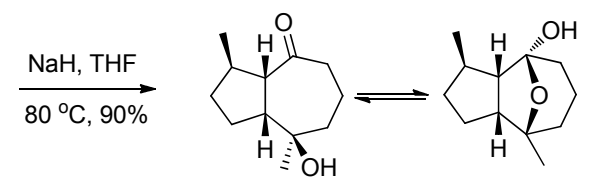

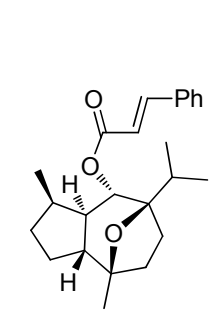

(-)-9-deoxy-englerin A (67)
65

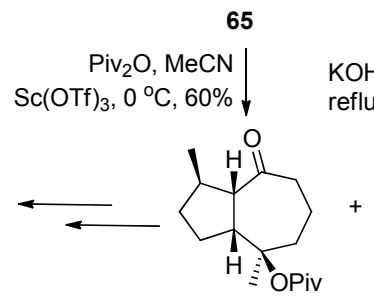

66
$65 a$
图式 14 (-)-9-脱氧-englerin A 的 Maier 合成路线

Scheme 14 Maier's synthesis of (-)-9-deoxy-englerin A

为起始原料, 经 Michael 加成得到化合物 74, 然后经 $\mathrm{SmI}_{2}$ 催化还原偶联一步构建 englerin A 氧杂三环骨架, 进而快速实现 englerin A 的形式合成. 整条合成线路只 有 8 步, 路线设计非常巧妙, 总收率高达 $20 \%$, 其合成 路线见 Scheme 15. 最近, Proter 小组也报道了利用 $\mathrm{SmI}_{2}$ 来还原内酯, 接着环化的串联反应来构建 5,7 元环骨架 (Eq. 1).

Yoshihiro 小组 ${ }^{[28]}$ 将铑催化的 4,6-二烯环合反应成 功地应用于天然产物 $( \pm)$-epiglobulol 全合成. 该研究小

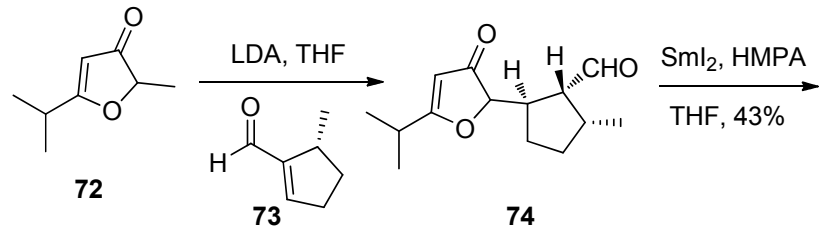

$75 \%, 2: 1 d r$

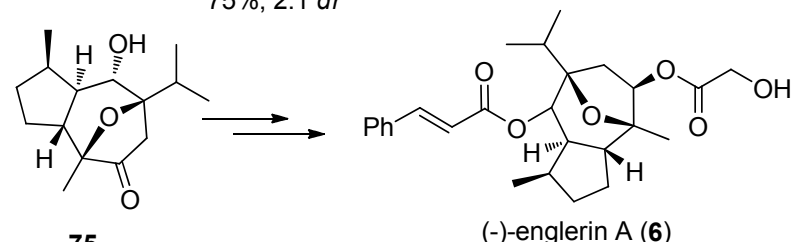

75

(-)-englerin A (6)

图式 15 (一)-englerin A 的 Chain 合成路线

Scheme 15 Chain's synthesis of (-)-englerin A

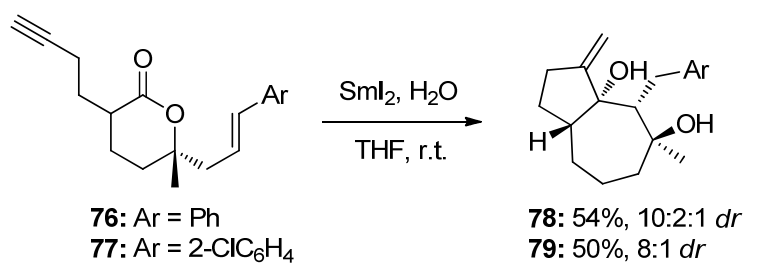

组首次发现铑催化 4,6-二烯 $(\mathbf{8 0})$ 分子内还原酰化反应, 以较好的收率得到环合产物 81. 进一步研究发现, 金属 铑也能高效地催化 1,3-二烯同系物 82 分子内环合反应, 如 Scheme 16 所示.
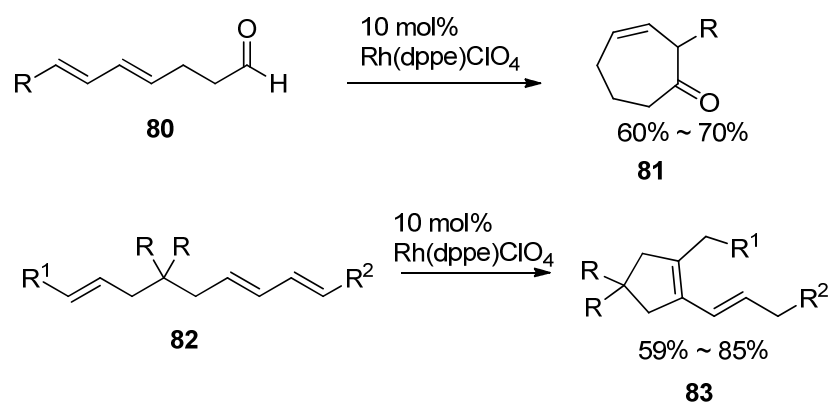

图式 16 铑催化的 4,6-二烯环合反应

Scheme 16 Rh-catalyzed cyclization of 4,6-diene

Yoshihiro 小组将该反应成功地应用于愈创木烷型 天然产物 5, 7 元环的构建. 化合物 84 在金属铑催化下发 生分子内的环合反应，顺利构建 5,7 元环基本骨架，以 44\%的收率得到环合产物 85 , 然后经多步反应完成天然 产物 $( \pm)$-apoaromadendrone (86) 和( \pm )-epiglobulol (87) 的全合成, 如 Scheme 17 所示.

\section{2 结束语}

综上所述，愈创木烷型倍半萜类天然产物是一类具 有重要生理活性的化合物, 本文简要介绍了近年来此类 天然产物的全合成研究进展, 即以卡宾羰基叶立德环化 串联反应、烯烃复分解环化反应、分子内羟醛缩合反应 
<smiles>[R]C=CC=CC(C=CC=CCCC=O)(C(C)=O)C(C)=O</smiles><smiles>C=CC[C@@H]1C(C(C)=O)CC2(CC(=O)OC)C(=O)CCC=C[C@H]12</smiles>

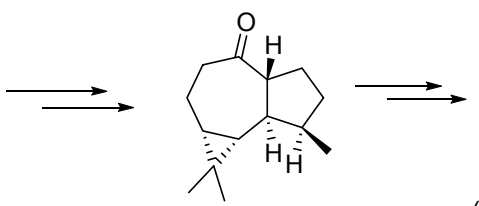

$( \pm$ )-apoaromadendrone (86)

( \pm )-epiglobulol (87)

图式 17 (土)-apoaromadendrone 和( \pm )-epiglobulol 的 Yoshihiro 合成路线

Scheme 17 Yoshihiro's synthesis of $( \pm)$-apoaromadendrone and ( \pm )-epiglobulol

等作为为关键反应的方法构建愈创木烷型倍半萜类天 然产物基本骨架. 然而, 如何简便、有效地构筑此类天 然产物碳骨架仍是具有挑战性的课题, 同时新方法的研 究对深入开展此类天然产物生物活性与构效关系的研 究具有重要的意义.

\section{References}

[1] (a) Connolly, J. D.; Hill, R. A. Dictionary of Terpenoids, Vol. 1, Chapman \& Hall, London, 1991, p. 476. (b) Fraga, B. M. Nat. Prod. Rep. 2011, 28, 1580.

[2] Zeng, J.-H.; Chen, X.; Pan, Y.-W. Chin. Herb. Med. 2008, 31, 168 (in Chinese). (曾建红，陈旭，潘艳薇等，中药材， 2008，31，168.)

[3] (a) Hikino, H.; Meguro, K.; Sakurai, Y.; Takemoto, T. Chem. Pharm. Bull. 1979, 27, 275.

(b) Inayama, S.; Gao, J. F.; Harimaya, K.; Kawamato, T.; Iitaka, Y.; Guo, Y. T. Chem. Pharm. Bull. 1984, 32, 3783.

(c) Hisashi, M.; Toshio, M.; Iwao, T.; Kiyofumi, N.; Masayuki, Y. Chem. Pharm. Bull. 2001, 49, 1558.

[4] Jiang, J.-P. Jilin Chin. Med. 2000, 2, 62 (in Chinese). (姜建萍, 吉林中医药, 2000, 2, 62.)

[5] (a) Irie, K.; Yoshioka, T. Nakai, A.; Ochiai, K.; Nishikori, T.; Wu, G.-R.; Shibuya, H.; Muraki, T. Eur. J. Pharmacol. 2000, 403, 235.

(b) Yoshioka, T.; Fujii, E.; Endo, M.; Wada, K.; Tokunaga, Y.; Shiba, N.; Hohsho, H.; Shibuya, H.; Muraki, T.; Inflammation Res. 1998, 47, 476.

(c) Ozaki, Y. Chem. Pharm. Bull. 1990, 38, 1045.

(d) Jang, M. K.; Sohn, D. H.; Ryu, J.-H. Planta Med. 2001, 67, 550.

[6] (a) Zhou, B. N. In Phytochemistry of Plants Used in Traditional Medicine, Eds.: Hostettmann, K.; Marston, A.; Maillard, M.; Hamburger, M., Clarendon Press, Oxford, 1995, pp. 313 334 .

(b) Yao, J. X.; Lin, X. Y. Acta Chim. Sinica 1982, 40, 385.

[7] Gong, X.; Wang, M.; Zhen; Tashiro, W. S.; Onodera, S.; Ikejima, T. Exp. Mol. Med. 2004, 36, 551.

[8] Maimone, T. J.; Baran, P. S. Nat. Chem. Biol. 2007, 3, 396.
[9] Ratnayake, R.; Covell, D.; Ransom, T. T.; Gustafson, K. R.; Beutler, J. A. Org. Lett. 2009, 11, 57.

[10] (a) Chain W. J. Synlett 2011, 2605.

(b) Pouwer, R. H.; Richard, J. A.; Tseng, C. C.; Chen, D. Y.-K. Chem. Asian J. 2012, 7, 22.

(c) Yue, G. Z.; Huang, Q. M.; Zou, P. Prog. Chem. 2012, 24, 1742 (in Chinese). (乐贵洲, 黄乾明, 邹平, 化学进展, 2012, 24, 1742.)

[11] Padwa, A. Tetrahedron 2011, 67, 8057.

[12] Geng, Z.; Chen, B.; Chiu, P. Angew. Chem., Int. Ed. 2006, 45, 6197.

[13] Navickas, V.; Ushakov, D. B.; Maier, M. E.; Strobele, M.; Meyer, H. J. Org. Lett. 2010, 12, 3418.

[14] (a) Amstrong, S. K. J. Chem. Soc., Perkin Trans. 1 1998, 371.

(b) Grubbs, R. H.; Chang, S. Tetrahedron 1998, 54, 4413.

(c) Furstner, A. Angew. Chem., Int. Ed. 2000, 39, 3013.

[15] Willot, M.; Radtke, L.; Konning, D.; Frohlich, R.; Gess-ner, V. H.; Strohmann, C.; Christmann, M. Angew. Chem., Int. Ed. 2009, 48, 9105.

[16] (a) Caine, D. In Comprehensive Organic Synthesis; Vol. 3, Eds.: Trost, B. M.; Fleming L.; Heathcock, C. H., Pergamon Press, New York, 1991, Chapter 1.1, pp. 1 110.

(b) Kadota, I.; Sakaihara, T.; Yamamoto, Y. Tetrahedron Lett. 1996, 37, 3195.

(c) Evans, D. A.; Bartroli, J.; Shih, T. L. J. Am. Chem. Soc. 1981, 103, 2127.

(d) Ito, Y.; Sawamura, M.; Hayashi, T. J. Am. Chem. Soc. 1986, 108,6405 .

(e) Corey, E. J.; Imwinkelried, R.; Pikul, S.; Xiang, Y. B. J. Am. Chem. Soc. 1989, 111, 5493.

(f) Abiko, A.; Liu, J. F.; Masamune, S. J. Am. Chem. Soc. 1997, 119, 2586.

(g) Kadota, I.; Park, J. Y.; Yamamoto, Y. J. Chem. Soc., Chem. Commun. 1996, 841.

[17] Nicolaou, K. C.; Kang, Q.; Ng, S. Y.; Chen, D. Y.-K. J. Am. Chem. Soc. 2010, 132, 8219.

[18] Xu, J.; Caro-Diaz, E. J. E.; Theodorakis, E. A. Org. Lett. 2010, 12, 3708.

[19] Zhou, Q.; Chen, X.; Ma, D. Angew. Chem., Int. Ed. 2010, 49, 3513.

[20] Jimenez-Nenez, E.; Molawi, K.; Echavarren, A. M. Chem. Commun. 2009, 47, 7327.

[21] Molawi, K.; Delpont, N.; Echavarren, A. M. Angew. Chem., Int. Ed. 2010, 49, 3517.

[22] Williams, M. J.; Deak, H. L.; Snapper, M. L. J. Am. Chem. Soc. 2007, 129, 486.

[23] Srikrishna, A.; Pardeshi, V. H.; Mahesh, K. Tetrahedron 2010, 21, 2830.

[24] Lee, E.; Yoon, C. H. Tetrahedron Lett. 1996, 37, 5929.

[25] (a) Sun, B. F.; Wang, C. L.; Ding, R.; Xu, J. Y.; Lin, G. Q. Tetrahedron Lett. 2010, 52, 2155.

(b) Wang, C. L.; Sun, B. F.; Chen, S. G.; Ding, R.; Lin, G. Q.; Xu, J. Y. ; Shang, Y. J. Synlett 2012, 263.

[26] Ushakov, D. B.; Navickas, V.; Ströbele, M.; Maichle-Mössmer, C.; Sasse, F.; Maier, M. E. Org. Lett. 2011, 13, 2090.

[27] Li, Z.; Nakashige, M.; Chain, W. J. J. Am. Chem. Soc. 2011, 133, 6553.

[28] Yoshihiro, O.; Ai, T.; Miwako, M. Tetrahedron Lett. 2006, 47, 5617. 